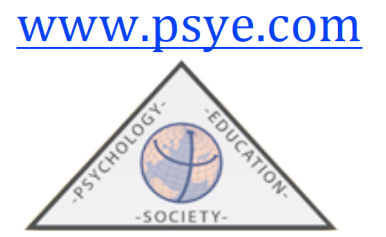

\title{
Desarrollo Profesional Docente en Singapur: Describiendo el Panorama
}

\author{
Alfredo BAUTISTA, Joanne WONG, \& Saravanan GOPINATHAN \\ Nanyang Technological University, National Institute of Education (Singapur)
}

(Recibido el 20 de enero 2015; Aceptado el 31 de agosto de 2015)

\begin{abstract}
RESUMEN: Este artículo describe el modelo de desarrollo profesional docente (DPD) en Singapur, uno de los países con mejor desempeño en educación a nivel mundial. Ofrecemos una visión general de los recursos disponibles para los aproximadamente 30.000 profesores de las 350 escuelas primarias y secundarias gestionadas por el Ministerio de Educación (MOE). Nos centramos en los tres principales proveedores de DPD: el Instituto Nacional de Educación, la Academia de Profesores de Singapur y los seis Centros de Excelencia, y las propias escuelas. Guiados por el "Modelo de Crecimiento Docente", el objetivo de dichos proveedores es fomentar un DPD coherente con los intereses de los profesores, con las necesidades de las escuelas, y el con el currículo nacional. Los profesores disponen de una asignación de DPD excepcionalmente alta (hasta 100 horas voluntarias anuales). Hay varios tipos de actividades en las que los docentes pueden participar, desde cursos formales y estructurados hasta iniciativas innovadoras de naturaleza informal (investigación-acción, "lesson study"). Profesores con diferentes niveles de experiencia y perfiles profesionales tienen acceso a diferentes oportunidades de DPD. La mayor parte focaliza en contenidos disciplinares específicos y proporciona a los profesores oportunidades para el aprendizaje colaborativo, el intercambio y la colaboración. De hecho, recientemente se ha requerido a todas las escuelas de Singapur adoptar el modelo de Comunidades de Aprendizaje Profesional (CAP). Concluimos que este abanico de recursos de DPD, considerados en conjunto, presenta las características del DPD de "alta calidad" descrito en la literatura internacional. Sin embargo, sugerimos que es necesaria más investigación que examine el grado en que este ambicioso modelo de DPD está mejorando el conocimiento y las prácticas docentes de los profesores, y en última instancia del aprendizaje de los alumnos.
\end{abstract}

Palabras clave: desarrollo profesional docente; Singapur, profesores en ejercicio, aprendizaje del profesor

\section{Teacher Professional Development in Singapore: Depicting the Landscape}

ABTRACT: This article depicts the landscape of teacher professional development (PD) in Singapore, one of the world's top-performing countries in education. We provide an overview of the resources available to the approximately 30,000 teachers within the 350 
primary and secondary schools run by the Ministry of Education (MOE). We focus on the three main PD providers: the National Institute of Education, the Academy of Singapore Teachers and six Centers of Excellence, and schools themselves. Guided by the "Teacher Growth Model," these providers aim at making PD coherent with teachers' interests, the needs of schools, and the national curriculum. Teachers in Singapore are given the exceptionally high allotment of 100 voluntary hours of PD per year. There are multiple types of activities teachers can engage in, ranging from formal/structured courses and programs to more informal/reform-based initiatives (action research, lesson study). Teachers with different levels of expertise and career paths have access to different PD opportunities. Most PD is subject-specific and provides teachers with opportunities for networked learning, collegial sharing, and collaboration. In fact, all MOE schools have been recently mandated to become Professional Learning Communities (PLC). We conclude that this comprehensive set of PD resources, considered as a whole, presents the features of "high-quality" PD described in the international literature. However, we suggest that more research is needed to examine the extent to which such an ambitious PD model is enhancing teachers' knowledge and pedagogies, and ultimately students' learning.

Keywords: teacher professional development, Singapore, in-service teachers, teacher learning

Agradecimientos: Este estudio fue financiado por el programa "Education Research Funding Programme, National Institute of Education (NIE), Nanyang Technological University, Singapore. Proyecto: OER 02/14 AA". Las opiniones expresadas en este artículo son de los autores y no representan necesariamente la opinión del National Institute of Education (NIE).

Correspondencia: Alfredo Bautista, Research Scientist \& Lecturer. Nanyang Technological University - National Institute of Education. Education \& Cognitive Development Lab. 1 Nanyang Walk. NIE5-B3-16. Singapur [637616]. Telf.: (+65) 6219 6256. Fax: (+65) 6896 9845. E-mail: Alfredo.Bautista@nie.edu.sg

\section{Cómo citar este articulo}

Bautista, A., Wong, J., \& Gopinathan, S. (2015). Desarrollo profesional docente en Singapur: Describiendo el Panorama (trad. al castellano de N. Navarro Gómez). Psychology, Society and Education, 7(3), 423-441. [V.O.: Teacher professional development in Singapore: Depicting the landscape. Psychology, Society and Education, 7(3), 311-326].

\section{Introducción}

Situada en el corazón del sudeste asiático, la pequeña ciudad-estado de Singapur es una historia fascinante de rápida transformación y éxito. Tras su independencia de Malasia en 1965, en solo tres décadas este país insular pasó de ser una aldea de pescadores a convertirse en un importante centro comercial, contando hoy día con uno de los mayores centros financieros del mundo. Inmediatamente después de su independencia, el gobierno unicameral a cargo del país (aún hoy en el poder) adoptó el inglés como principal medio de comunicación, no sólo para los negocios sino

\section{(C) Psy, Soc, \& Educ, 2015. Vol. 7(3)}


también en la educación. Viviendo en un país sin recursos naturales, los singapurenses consideran que la supervivencia de su nación está supeditada al desarrollo del "capital humano" (Gopinathan, 2012). Esta creencia se convirtió en una de las fuerzas impulsoras de los constantes esfuerzos de Singapur por mejorar la calidad de su sistema educativo, y en particular -como se muestra en este artículopor mejorar la preparación y oportunidades de desarrollo profesional que ofrece a su personal docente ${ }^{1}$ (Ellis, 2014).

Singapur es considerado actualmente como uno de los países con mejor desempeño en educación a nivel mundial, con base en indicadores como las puntuaciones de sus estudiantes en comparaciones internacionales, las tasas de graduación, y el porcentaje de estudiantes que cursan educación superior (Informe de Competitividad Global del Foro Económico Mundial 2014-2015, ver Schwab, 2015). Año tras año, los estudiantes de Singapur alcanzan las mejores puntuaciones en matemáticas, ciencias y lengua en evaluaciones internacionales como el "Programa para la Evaluación Internacional de Alumnos" (Programme for International Student Assessment - PISA), "Tendencias Internacionales en Matemáticas y Ciencias" (Trends in International Mathematics and Science Study - TIMSS), y el "Progreso en el Estudio Internacional de la Alfabetización y la Lectura" (Progress in International Reading Literacy Study - PIRLS).

Investigaciones recientes indican que el éxito del sistema educativo de Singapur está ligado a múltiples factores relativos a tres niveles independientes: el nivel macro (factores socio-culturales, económico-políticos); el nivel organizacional (calidad de las escuelas, de los profesores, planes de estudio, etc.); y el nivel familiar (crianza y socialización) (Dimmock y Tan, 2013; Hairon y Dimmock, 2011; Tan y Dimmock, 2014). Sin duda, el nivel organizacional es el que ha despertado mayor interés y curiosidad entre educadores, investigadores y responsables políticos de todo el mundo en los últimos años. El Ministerio de Educación de Singapur (Ministry of Education, o MOE por sus siglas en inglés) ha diseñado su sistema educativo mediante un cuidadoso examen de las "mejores prácticas" a nivel internacional (p.ej. escuelas exitosas, nuevas políticas educativas, nuevos currículos, programas de formación del profesorado, programas de desarrollo profesional y de liderazgo). Ello ha contribuido a transformar radicalmente el panorama educativo de Singapur en un corto lapso de tiempo (Gopinathan, 2012). Dado su innegable éxito, pensamos que otros países podrían estar interesados en analizar las prácticas actuales de Singapur en materia de educación.

Este artículo se centra en el Desarrollo Profesional Docente (DPD), que puede ser considerado como un factor específico dentro del nivel organizacional mencionado anteriormente. Hoy en día, Singapur ofrece una amplia variedad de oportunidades de desarrollo profesional a los profesores de todas las áreas y niveles

\footnotetext{
${ }^{1}$ En este artículo, se utiliza el término "profesores" para referirse de forma genérica a maestros y profesores, tanto de género masculino como femenino.
} 
educativos, desde educación infantil hasta educación superior. En este trabajo, ofrecemos una breve panorámica de los recursos disponibles para los aproximadamente 30.000 docentes que trabajan en las 350 escuelas primarias y secundarias gestionadas y subvencionadas por el $\mathrm{MOE}^{2}$. Se muestra que el DPD en este país (a) se centra en contenidos específicos y está conectado con la realidad del aula; (b) es intensivo y constante; (c) proporciona a los profesores oportunidades para el aprendizaje activo; (d) promueve la participación colectiva entre los profesores de distintas escuelas, y sobre todo dentro de la misma escuela; y (e) es coherente con las necesidades e intereses de los profesores y con las prioridades escolares y nacionales. Con base en estas características generales, argumentamos que el modelo de DPD en Singapur presenta las características del DPD de "alta calidad" descritas en la literatura internacional (p.ej., Avalos, 2011; Bautista, Cañadas, Brizuela, y Schliemann, 2015; Borko, 2004; Darling-Hammond y McLaughlin, 2011; Desimone, 2009). No obstante, dado que reconocemos la complejidad de razones que explican el éxito de Singapur en materia educativa (Dimmock y Tan, 2013; Hairon y Dimmock, 2011; Tan y Dimmock, 2014), nuestra intención no es en modo alguno establecer o sugerir relaciones causales simples entre la calidad y/o cantidad de oportunidad de DPD ofrecidas a los profesores y el alto rendimiento de sus estudiantes. Como se discute en la Introducción de este monográfico (Bautista y Ortega-Ruiz, 2015), la influencia del DPD debe ser analizada desde una perspectiva sistémica y compleja.

\section{Características Generales del Desarrollo Profesional Docente (DPD) en Singapur}

La profesión docente es altamente considerada y respetada en Singapur. Los ciudadanos comprenden que los profesores desempeñan un rol vital en el desarrollo y en el progreso de su nación. En consecuencia, los candidatos reclutados para convertirse en futuros profesores son elegidos muy cuidadosamente.

El MOE ha desarrollado estrategias variadas para la contratación, compensación, e inducción de los profesores de escuelas primarias y secundarias (Darling-Hammond, 2010). Los candidatos aceptados en programas de formación docente son seleccionados de entre aquellos alumnos con mejor rendimiento académico de cada cohorte (dentro del tercio superior). La mayoría de los candidatos han superado el examen del Cambridge Advanced Level (también conocidos como "A levels"), actualmente la evaluación más exigente en Singapur, puntuando al menos en la mitad del percentil superior. Los titulados universitarios son otros candidatos comúnmente reclutados para convertirse en profesores. Todo candidato ha de superar un proceso de selección que incluye la entrevista de un comité (centrada no sólo en las capacidades intelectuales del candidato, sino también en sus intereses y

\footnotetext{
${ }^{2}$ Nos centramos en profesores de escuelas primarias y secundarias gestionadas y subvencionadas por el MOE. Singapur también cuenta con algunas escuelas independientes, especializadas e independiente-especializadas (15 en total), así como un alto número de escuelas privadas que ofrecen programas educativos extranjeros (aproximadamente 40). Las oportunidades de DPD ofrecidas a los profesores de estas otras escuelas pueden diferir de las descritas en este artículo.
} 
cualidades personales), una evaluación de su compromiso con la profesión docente, su servicio a diversas poblaciones estudiantiles, sus resultados académicos y las contribuciones realizadas a su alma mater y a la comunidad. Tras este riguroso proceso de selección, que refleja el gran prestigio del que goza la profesión docente en Singapur, sólo una octava parte de los candidatos son aceptados (DarlingHammond, 2010). Una vez comienzan sus programas de formación inicial, los estudiantes reciben un salario equivalente al $60 \%$ del salario de un profesor en ejercicio durante la duración completa de sus programas (que oscilan entre los $3 \frac{1}{2}$ y los 6 años). Tras la finalizar sus estudios, se exige que los profesores ejerzan la profesión docente durante un determinado número mínimo de años, dependiendo de la duración de sus programas de formación inicial.

Con la iniciativa "Escuelas Pensantes, Nación que Aprende" (Thinking Schools, Learning Nation - véase Goh, 1997), Singapur comenzó a prestar especial atención al DPD ofrecido a su cuerpo de profesores. Esta iniciativa se alejó de la concepción tradicional del profesor como un mero técnico, pasando a concebir a los profesores como profesionales reflexivos (Deng, Gopinathan y Lee, 2013). Desde entonces, el DPD ha sido considerado como uno de los pilares esenciales para ayudar a los profesores a mejorar sus prácticas de enseñanza. Iniciativas posteriores puestas en marcha por el MOE, como el "Enseña Menos, Aprende Más" (Teach Less, Learn More - MOE, 2005) o el actual modelo de DPD, llamado "Modelo de Crecimiento Docente" (Teacher Growth Model - MOE, 2012), han puesto de relieve aún más el papel clave que el DPD juega en la mejora de la calidad de la educación. Actualmente, los responsables políticos, investigadores y educadores de docentes están de acuerdo en que las reformas de innovación educativa requieren de políticas de DPD, y en que apoyar la construcción de las capacidades del profesorado es esencial para que las reformas sean exitosas.

El "Modelo de Crecimiento Docente" anima a los profesores a profundizar en su aprendizaje a través de una variedad de formatos y plataformas, incluyendo cursos presenciales y online, talleres y programas de postgrado (p.ej., Masters y Doctorados), conferencias profesionales, convenciones y simposios, investigaciónacción, tutorías y coaching, así como mediante asociaciones escuela-universidad (MOE, 2012). Las diferentes iniciativas abarcan desde formas tradicionales de DPD (como cursos formales) hasta iniciativas basadas en los principios de las recientes reformas educativas (como sesiones informales de intercambio, investigación-acción, observaciones por pares, o "lesson study"). También se anima a los docentes a utilizar parte de su tiempo de DPD para aprender sobre temas que no están directamente relacionadas con la enseñanza, como por ejemplo sobre competencias terapéuticas, educación multicultural, bienestar personal, o habilidades sociales (Tripp, 2004). La justificación radica en que, a través de este tipo de experiencias, los profesores desarrollarán nuevas herramientas para satisfacer las necesidades y los desafíos de la educación del siglo XXI (MOE, 2012). 
Los profesores disponen de hasta 100 horas de actividades de DPD por año. Completar estas 100 horas es un derecho (opcional), no un requisito, aunque la mayoría de profesores se benefician de estas oportunidades (Wong, 2013). Cada año, al menos uno de los miembros de la junta directiva escolar (generalmente compuesta por el director, el subdirector, los jefes de departamento/materia, y en algunos casos un responsable de recursos humanos y desarrollo profesional, llamado staff developer) discute con los profesores del centro cómo planear su agenda anual de $\mathrm{DPD}$, en respuesta a las motivaciones e intereses de cada profesor, de las necesidades de la escuela, y de los requerimientos del currículo nacional. El DPD de cada profesor ha de ser aprobado por su respectivo supervisor y ha de ser relevante para su rol y ámbito de trabajo. Las escuelas tienen sus propias políticas respecto a lo que constituye DPD relevante y respecto al proceso de aprobación. El costo asociado con el DPD está subvencionado por el MOE en su totalidad (Wang, Kim, Lee y Kim, 2014).

Los resultados de la "Encuesta Internacional de Enseñanza y Aprendizaje" (Teaching and Learning International Survey, TALIS) de 2013, realizada por la Organización para la Cooperación y el Desarrollo Económico (OCDE, 2014), han mostrado que las tasas de participación en actividades de DPD son muy superiores en Singapur que en otros países. Por ejemplo, en comparación con los 34 países que participaron en el estudio, mayores proporciones de profesores singapurenses de secundaria participaron en cursos y talleres de formación $(93 \%$ de los profesores en Singapur, en comparación con el promedio de $71 \%$ ), en tutorías, observación entre pares y coaching (65\% en Singapur, en comparación con el $29 \%$ ), en seminarios y conferencias (61\% en comparación con el $44 \%)$, en experiencias de aprendizaje en red (53\% frente a 37\%), y en investigaciones llevadas a cabo en la escuela, tanto a nivel individual como en colaboración (45\% en comparación con el 31\%).

El DPD en Singapur tiene como objetivos ofrecer a los profesores oportunidades de aprendizaje que se ajusten a sus necesidades y que respondan a sus motivaciones y metas personales. Para alcanzar dichos objetivos, el "Modelo de Crecimiento Docente" (MOE, 2012) conceptualiza el DPD como un continuo que comienza con la preparación inicial y la inducción, el desarrollo y crecimiento continuo, y que incluye programas que marcan los distintos hitos de la carrera profesional docente (Chong y Fong, 2000). Así, en función del nivel de experiencia de los profesores (principiante, avanzado, experto), típicamente se les ofrece diferentes "rutas". En muchas escuelas, existe un responsable de recursos humanos y desarrollo profesional (o staff developer), quien se encarga de asegurar la coherencia entre los objetivos profesionales de los profesores y las oportunidades de DPD que se les ofrecen, proporcionándoles DPD que se adapte a sus competencias e intereses y asignándoles distintos roles dentro del sistema educativo (Hairon y Dimmock, 2011). El estudio TALIS también ha indicado que las escuelas de Singapur tienen una arraigada cultura de mentoría, teniendo la mayor proporción de profesores que actúan como mentores de sus compañeros más jóvenes $(39 \%$ de los docentes, en comparación con el promedio de 14\%). 
Otra importante estrategia del modelo de DPD de Singapur consiste en ofrecer a los profesores tres diferentes itinerarios profesionales, denominados itinerario de enseñanza (siendo "Profesor-Maestro Principal" su posición más alta), el itinerario de liderazgo (cuyo papel más alto es el "Director General de Educación"), y el itinerario de especialista (cuyo máximo rol es el de "Jefe Especialista", donde los profesores centran en política educativa e investigadora) (Tan, Wong y Goh, 2010). Esta estrategia fue desarrollada para responder a los objetivos profesionales de los docentes y para fomentar el desarrollo del talento y la capacidad de manera sistemática.

\section{Los Tres Principales Proveedores de DPD en Singapur}

En Singapur, existen tres principales proveedores de DPD para los profesores de primaria y secundaria. Uno de ellos es el Instituto Nacional de Educación (National Institute of Education, NIE), que es el principal proveedor de cursos y programas de postgrado en educación para profesores noveles, en liderazgo instruccional para los profesores más sénior, y en liderazgo educativo para jefes de departamento, subdirectores y directores. El segundo proveedor es la Academia de Profesores de Singapur (Academy of Singapore Teachers, AST), establecida por el MOE en 2010. La AST reúne a profesores de diferentes escuelas y les proporciona oportunidades de aprendizaje colaborativo. Las escuelas pueden ser consideradas como el tercer proveedor principal de DPD, ya que muchas oportunidades de aprendizaje del profesorado se contextualizan dentro de cada escuela particular. De hecho, Singapur pone mucho énfasis en las formas de DPD colaborativo y orientado-a-la-comunidad, pues se consideran formas potentes para ayudar a los profesores a mejorar no sólo sus conocimientos disciplinares y pedagógicos, sino también para fomentar su sentido de pertenencia, de camaradería y la solidaridad entre los profesores.

El "Modelo de Colaboración Mejorado" (Enhanced Partnership Model) subraya la fuerte relación tripartita entre estos tres proveedores de DPD (NIE, AST y las escuelas), lo que garantiza la coherencia y el rigor de las diferentes oportunidades que se ofrecen a los profesores (véase NIE, 2009). A continuación, describimos los enfoques de DPD adoptados por estos tres proveedores, aportando algunos ejemplos ilustrativos.

\section{Instituto Nacional de Educación (NIE)}

El Instituto Nacional de Educación (National Institute of Education, o NIE por sus siglas en inglés), es la única institución que ofrece programas de formación inicial del profesorado en Singapur, así como uno de los principales proveedores del DPD para profesores en ejercicio y otros agentes educativos (p.ej., directivos escolares, especialistas curriculares, profesores investigadores). Desde la década de 1970, el NIE ha proporcionado las oportunidades de DPD requeridas por el cuerpo docente de Singapur, en estrecha coordinación con el MOE (Tan et al., 2010). Por ejemplo, para hacer frente a la escasez de profesores especializados en música y artes visuales en 
escuelas primarias, el NIE ha venido ofreciendo desde 2011 un Diploma Avanzado en Educación Musical Primaria. Asimismo, el NIE está comprometido con el diseño de DPD que contemple las necesidades de aprendizaje reales del profesorado, dotándolo de nuevas competencias en respuesta a las necesidades y demandas de la sociedad actual.

El NIE actualmente ofrece una amplia variedad de cursos independientes de DPD para profesores de las diferentes materias. Los cursos se centran principalmente en contenidos específicos, desarrollo curricular, pedagogía, evaluación y aprendizaje del alumno. La mayoría de estos cursos cortos conducen a la obtención de diplomas y/o cualificaciones profesionales avanzadas. Por ejemplo, el Diploma Avanzado en Educación Musical Primaria citado anteriormente comprende siete cursos, divididos en conocimiento del contenido musical (teoría de la música, músicas del mundo), habilidades musicales (destrezas instrumentales, dirección, actuación), y estudios curriculares (teoría y práctica de la educación musical). Dicho diploma permite a los profesores de música de primaria acceder a otras titulaciones especializadas, incluyendo estudios superiores de grado y posgrado. Con el fin de evaluar el impacto de este tipo de programas, el NIE a menudo ofrece a sus investigadores proyectos para analizar los cambios en los conocimientos y en las prácticas de aula de los docentes. Por ejemplo, Costes-Onishi y su equipo están evaluando actualmente la eficacia del Diploma Avanzado en Educación Musical Primaria (Costes-Onishi y Caleon, 2014). Además, como se mencionó anteriormente, el NIE es el principal proveedor de programas de postgrado en educación (p.ej., Masters en Enseñanza, Masters de Educación, Doctor en Educación), en liderazgo instruccional (p.ej., el Programa de Profesores Sénior), y en liderazgo educativo (p.ej., Gestión y Liderazgo de Escuelas). El MOE ofrece numerosas becas para profesores interesados en realizar estudios de postgrado en Singapur y en el extranjero, ya sea a tiempo completo o parcial $^{3}$.

La metodología de los cursos y programas de DPD en NIE incluye clases magistrales de especialistas, talleres prácticos, trabajo en proyectos y de campo, investigación-acción, y un amplio rango de actividades que requieren tanto trabajo individual como grupal. Para completar estos cursos, los profesores generalmente necesitan interactuar entre sí tanto de forma presencial como online. Una vez finalizado el curso, se otorga a los profesores o bien un certificado de asistencia o un certificado de aprovechamiento, en función de los requerimientos específicos. El certificado de aprovechamiento requiere que, además de asistir al curso, el profesor haya alcanzado un nivel mínimo de rendimiento.

El NIE colabora en numerosos proyectos de pequeña escala con escuelas interesadas en mejorar aspectos específicos de tipo curricular y/o pedagógico (Wang et al., 2014). Por otra parte, el NIE produce diversas publicaciones para promover aún más el aprendizaje continuo de los docentes. Por ejemplo, resúmenes de los numerosos proyectos de investigación realizados por el personal docente e investigador del NIE se comparten periódicamente con toda la comunidad educativa.

\footnotetext{
${ }^{3}$ Visitar: http://www.nie.edu.sg/studynie/professional-development-programmes-and-courses/

(C) Psy, Soc, \& Educ, 2015. Vol. 7(3)
} 
La información publicada en el sitio web del NIE pone especial énfasis en los resultados e implicaciones de los estudios realizados. Se cuida que la información utilice un lenguaje accesible (p.ej., evitando la jerga técnica). La intención es maximizar el impacto de la investigación en el conocimiento y la práctica de aula de los docentes. Existen otras tres publicaciones internas donde el NIE comparte sus resultados de investigación con los profesores locales y los diferentes agentes educativos: 1) SingTeach, una revista electrónica dirigida a profesionales de la educación y que publica informes escritos por profesores; 2) NIE Research Brief Series, está principalmente dirigida a los responsables políticos, autoridades escolares, administradores e investigadores; y 3) ReED (Research in Education) Bulletin, cuya audiencia es la comunidad educativa a nivel más global ${ }^{4}$.

\section{Academia de Profesores de Singapur (AST) y los Seis Centros de Excelencia}

El segundo principal proveedor de DPD es la Academia de Profesores de Singapur (Academy of Singapore Teachers, o AST por sus siglas en inglés). Fue establecida en 2010 junto con seis Centros de Excelencia de DPD. Las Academias reúnen a profesores de diferentes escuelas y les involucra en múltiples actividades de aprendizaje colaborativo. Más específicamente, las Academias fueron diseñadas para facilitar que los profesores discutan y compartan prácticas pedagógicas innovadoras, elevando de este modo los estándares de calidad en las distintas disciplinas y fomentando una robusta cultura de intercambio profesional y colaboración, liderada por los propios profesores (MOE, 2010).

La AST ha continuado el trabajo de vanguardia iniciado en 1998 por la "Red de Docentes" (Teachers' Network) del MOE, que fue pionera en abogar por la necesidad e importancia del DPD dirigido y liderado por el profesorado (Hairon y Dimmock, 2011). Siguiendo las tendencias observadas en ciertas escuelas anglosajonas, la Red de Profesores diseñó e implementó una serie de programas de DPD interconectados, que facilitaban la colaboración de los profesores, la reflexión y la investigación (Tripp, 2004). La plataforma clave de aprendizaje era conocida como "Círculos de aprendizaje", donde pequeños grupos de profesores auto dirigidos llevaban a cabo actividades como investigación-acción o "lesson study". A partir del conocimiento construido dentro de estos círculos de aprendizaje, los profesores se implicaban después en otros programas como talleres organizados por profesores, conferencias, páginas webs y publicaciones. El lema "Para los Profesores, Por los Profesores" (For Teachers, By Teachers - MOE, 2005) refleja la filosofía del aprendizaje y el cambio docente mantenida por la Red de Docentes. Las políticas e iniciativas educativas posteriores a 1998 han continuado proporcionando a los profesores agencia y responsabilidad plena respecto a su desarrollo profesional. La justificación radica en que los profesores no deben ser meros "receptores" o "ejecutores" de las ideas discutidas en DPD, sino verdaderos "productores" de

\footnotetext{
${ }^{4}$ Visitar: http://www.nie.edu.sg/office-education-research/publications/
} 
conocimiento, asumiendo las riendas de su propio aprendizaje continuo (Ellis y Armstrong, 2013; Hairon y Dimmock 2011; Wong, 2013). La Academia fue creada con el propósito de crear esta fraternidad de los profesores activos, comprometidos y reflexivos, y para facilitar la creación de comunidades de aprendizaje entre los profesores.

La AST incluye cuatro áreas de especialización, llamadas Subject Chapters, que pueden conceptualizarse como comunidades de aprendizaje de contenidos específicos. Cada Subject Chapter está dividido en asignaturas escolares concretas (Tabla 1). Todos los profesores del MOE están invitados a participar en las actividades que se organizan, lo que les proporciona numerosas oportunidades para aprender con/de otros colegas. De acuerdo al sitio $w^{5} b^{5}$ de la AST, alrededor de 3.500 profesores participaron en las actividades organizadas a través de los Subject Chapters en 2014.

Tabla 1. Áreas de especialización (Subject Chapters) de la Academia de Profesores de Singapur

\begin{tabular}{cccc}
\hline Humanidades & Matemáticas & Ciencias & Otras \\
\hline Geografía & Matemáticas de Primaria & Biología & Diseño \& Tecnología \\
Historia & Matemáticas de Secundaria & Química & Nutrición \& Ciencias de la \\
& & comida \\
Estudios sociales & & Física & Principios de Contabilidad \\
& & Ciencias de & \\
& & Primaria & \\
\hline
\end{tabular}

Además de los Subject Chapters, existen seis Centros de Excelencia (también conocidos como Academias o Institutos). Cuatro de ellos ofrecen cursos de DPD a los profesores de las diferentes lenguas enseñadas en las escuelas singapurenses, es decir, el inglés, que es el principal medio de instrucción (English Language Institute of Singapore), el chino mandarín (Singapore Center for Chinese Language), el malayo (Malay Language Center of Singapore) y el tamil (Umar Pulavar Tamil Language Center). Los otros dos centros se especializan en DPD de Música y Artes (Singapore Teachers' Academy for the Arts) y de Educación Física (Physical Education \& Sports Teacher Academy).

Los Subject Chapters y los Centros de Excelencia organizan una amplia variedad de actividades de DPD, que van desde actividades formales (p.ej., talleres de métodos de investigación escolar, cursos y seminarios centrados en contenidos disciplinares específicos y conocimientos pedagógicos, conferencias y simposios) hasta actividades de DPD de carácter más informal e innovador (p.ej., la investigación-acción, discusiones grupales). El aprendizaje de los profesores es apoyado por "Un Portal Para Todos" (One Portal All Learners, OPAL por sus siglas en inglés), una plataforma online con varios repositorios de gestión de contenidos que contiene materiales de enseñanza/aprendizaje y numerosa información útil para los profesores.

\footnotetext{
${ }^{5}$ Visitar: http://www.academyofsingaporeteachers.MOE.gov.sg/

(C) Psy, Soc, \& Educ, 2015. Vol. 7(3)
} 
Veamos algunos ejemplos de actividades de DPD realizadas recientemente por las Academias de profesores. Una serie de talleres sobre "lesson study" ejemplificaría las actividades de DPD de corte más estructurado o formal. Estos talleres se dirigieron a profesores de ciencias de primaria y secundaria, con el objetivo de apoyar sus procesos de reflexión y discusión en sus respectivas escuelas (Heong, 2012). Otro ejemplo es el "Programa Estructurado de Mentoría", donde profesores principiantes reciben apoyo y orientación de los colegas con más experiencia durante su inducción en la profesión docente (Wong, 2013).

Respecto a actividades de DPD de tipo más informal, las "Redes de Profesores-Investigadores" constituyen una de las iniciativas más interesantes. Estas redes son comunidades de aprendizaje integradas por personal investigador del NIE, especialistas del MOE y profesores-investigadores, incluyendo a aquellos con mayor entrenamiento formal en investigación (denominados "activistas de investigación"). El objetivo general de estas comunidades de aprendizaje es capacitar a los docentes para realizar proyectos de investigación-acción (Hairon, 2006), una forma de específica investigación escolar donde los profesores discuten y reflexionan sobre problemas pedagógicos y en la que encuentran sus propias soluciones para mejorar la enseñanza y el aprendizaje. La investigación-acción requiere que los profesores observen de forma sistemática situaciones de enseñanza y aprendizaje. También requiere la recogida de evidencia empírica, que será utilizada más tarde por el profesor-investigador para reflexionar, tomar decisiones y desarrollar pedagogías más eficaces. Los ciclos de investigación-acción generalmente siguen la estructura de un procedimiento de resolución de problemas, que se componen de cinco pasos:

1. Identificación y definición del problema;

2. Planificación para la mejora;

3. La ejecución de las actividades de enseñanza / aprendizaje;

4. Observación de resultados (recolección de datos);

5. Reflexión sobre los resultados.

Una vez completado el ciclo de investigación-acción, el grupo de participantes escribe un diario reflexivo donde se resumen los procedimientos, resultados, conclusiones e implicaciones de su estudio. Normalmente, estos diarios de investigación-acción son compartidos con otros grupos de profesores interesados en problemas de aula similares. La AST facilita foros donde los profesores pueden compartir los resultados de su investigación, incluyendo un simposio anual y diversas publicaciones (Ellis, 2014). El proceso de investigación-acción y la colaboración con los diferentes miembros de la red de profesores-investigadores generalmente ayudan a los docentes a mejorar sus competencias reflexivas e investigadoras. Con el apoyo de los directivos escolares, estas experiencias de DPD tienen gran potencial para capacitar a los profesores a liderar innovaciones pedagógicas y curriculares en las escuelas.

\section{DPD en la escuela: Comunidades de Aprendizaje Profesional (CAPs)}


Gran parte del desarrollo profesional de los docentes de primaria y secundaria en Singapur se produce dentro de las propias escuelas, donde se ofrecen numerosas oportunidades de aprendizaje contextualizadas en el contexto de trabajo. De hecho, se entiende que las escuelas deben ser las principales organizaciones que promueven el aprendizaje de los profesores, y que éstas deben ser concebidas como “organizaciones que aprenden". En 2009/10, tras más de una década de iniciativas innovadoras de DPD, el MOE estableció que todas las escuelas públicas debían convertirse en Comunidades de Aprendizaje Profesional (CAP). Esta medida (ver MOE, 2010) convirtió a Singapur en el primer país del mundo en adoptar el marco de CAP a nivel nacional (Dimmock y Tan, 2013; Hairon y Dimmock, 2011).

Encabezados por los líderes escolares (p.ej., los responsables de recursos humanos y desarrollo profesional, los jefes de departamento, los especialistas en contenido), y siempre con el apoyo de la Academia de Profesores de Singapur, las CAP proporcionan a los profesores de Singapur estructuras y recursos para participar en gran variedad de prácticas de DPD basadas en la investigación, tales como investigación-acción, "lesson study" y una amplia gama de círculos de aprendizaje centrados en diferentes temáticas (p.ej., innovación curricular, prácticas docentes centradas en el estudiante, nuevos usos de las TICs, planificación de docencia colaborativa y de aprendizaje basado en proyectos). A estos grupos de colegas que aprenden y colaboran juntos se les conoce como "Equipos de Aprendizaje". Por lo general, estos equipos suelen completar su trabajo durante intervalos de tiempo denominados "espacios en blanco" (Wong, 2013). Se recomienda que las escuelas de MOE proporcionen al menos una hora de tiempo curricular a la semana para que los profesores participen en este tipo de iniciativas internas de DPD. Los equipos de aprendizaje comúnmente planifican alrededor de 8-10 sesiones de dos horas de duración, distribuidas a lo largo de todo el año académico. Estas horas de trabajo son reconocidas en la evaluación anual de los docentes, pudiéndose incluir dentro de las 100 horas anuales de formación optativa.

Una de las prácticas más extendidas dentro de las CAP en Singapur es la investigación-acción (Hairon, 2006; Wong, 2013), que fue descrita anteriormente. Otro tipo de DPD que ha cobrado importancia es modelo japonés "lesson study" (Lewis, Perry y Hurd, 2004), que fue introducido en las escuelas de Singapur en 2005. Al igual que en la investigación-acción, el objetivo general de "lesson study" es fomentar entre los docentes la investigación colaborativa y la reflexión pedagógica basada en evidencia empírica. Pese a las múltiples variaciones de este enfoque en la literatura, los profesores de Singapur generalmente adoptan modelos estándar compuestos por cuatro fases cíclicas y recurrentes (Tan, 2014):

1. Fase de estudio, cuando los profesores los contenidos de enseñanza y definen los objetivos de aprendizaje a largo plazo;

2. Fase de planificación, en la cual los profesores seleccionan las lecciones de investigación, predicen las ideas y dificultades de los estudiantes, y planifican la ejecución de metodologías específicas para la recopilación de datos; 
3. Fase de análisis, cuando los profesores observan y discuten la evidencia recopilada en el aula (p.ej., mediante videos, o analizando el trabajo escrito de los estudiantes);

4. Fase de reflexión, durante la cual los profesores discuten el aprendizaje de los estudiantes e identifican nuevas áreas para futuras investigaciones.

El MOE ha realizado grandes esfuerzos para apoyar el éxito de la iniciativa de las CAP. Por ejemplo, el MOE elaboró un manual (llamado "Kit de Iniciación CAP", en inglés PLC Starter Kit) explicando los beneficios de las CAP, las funciones desempeñadas por los diferentes miembros, y la metodología, procedimientos y herramientas que se pueden utilizar al realizar investigaciones dentro del aula (TDD, 2010). Por otra parte, el MOE proporciona fondos para que uno o varios profesores en cada escuela puedan convertirse en "activistas de investigación", con la expectativa de que ayuden a sus compañeros en la realización de investigaciones en el aula ${ }^{6}$ (Ellis, 2014). También se espera que dediquen parte de su tiempo a realizar trabajos de desarrollo, proponiendo innovaciones curriculares y realizando investigaciones escolares de vanguardia, con potenciales implicaciones para a nivel político y práctico. En 2009, se llevó a cabo una experiencia piloto de la iniciativa de CAP, únicamente con 51 escuelas. Alrededor de 2013, prácticamente todas las escuelas del MOE en Singapur ya habían abordado el esquema de las CAP (Lee, Hong, Tay y Lee, 2013).

\section{Direcciones Futuras}

Resulta evidente que medir el impacto de un modelo de DPD tan comprehensivo es una tarea complicada. Como se explicó anteriormente, los profesores en Singapur diseñan sus propias rutas de DPD en consulta con los líderes escolares. Por ello, dada la variedad y diversidad de actividades en las que potencialmente pueden participar cada año, la realización de estudios cuantitativos a gran escala que midan la efectividad de los programas de DPD en Singapur sería altamente compleja, ya que el número de variables no controladas y extrañas sería elevado. Sin embargo, pese a las dificultades metodológicas, sugerimos que es necesaria más investigación que analice el grado en que este ambicioso modelo de DPD está mejorando el conocimiento y las pedagogías de los profesores, y en última instancia del aprendizaje de los estudiantes. A nivel internacional, los investigadores en DPD han propuesto recientemente nuevas metodologías de investigación, tanto cuantitativas (Hill, Beisiegel y Jacob, 2013) como cualitativas (Kazemi y Hubbard, 2008), que se podrían aplicarse en el contexto de Singapur.

\footnotetext{
${ }^{6}$ Además de la investigación-acción llevada a cabo por profesores internamente en las escuelas, hay algunos investigadores del NIE que también llevan a cabo una investigaciones independientes sobre el tema (véase Hairon, 2006; Tan, 2014). Los estudios disponibles hasta el momento, sin embargo, no se han centrado en la evaluación de la eficacia de esta forma de DPD con respecto a las prácticas instruccionales de los profesores y/o el aprendizaje de los estudiantes.
} 
Algunos estudios realizados por investigadores del NIE han investigado la eficacia de cursos/programas formales específicos de DPD y otros proyectos de colaboración universidad-escuela centrados en matemáticas (Kaur, 2011), en ciencias (Tan y Nashon, 2013), en artes y música (Costes-Onishi y Caleon, 2014), y en prácticas de evaluación de la alfabetización (Koh, 2011). La mayoría de la investigación publicada es cualitativa y se basa en números reducidos de participantes. Los estudios se han centrado principalmente en el aprendizaje de los profesores (cambios en sus creencias, conocimientos, habilidades, y en menor medida las prácticas de aula), pero rara vez en el aprendizaje de los estudiantes. En general, las experiencias de DPD descritas en estos estudios produjeron resultados positivos, desde cambios modestos a mejoras más significativas. Por el contrario, no existe mucha investigación publicada acerca de la eficacia del DPD de corte informal, como la investigación-acción o el "lesson study", pese a ser prácticas muy extendidas en Singapur. Ambas prácticas son comúnmente utilizadas para ayudar a los profesores a desarrollar sofisticadas habilidades de investigación (Wong, 2013). Dado que ambas requieren enormes recursos en términos de tiempo y esfuerzo por parte de los docentes, consideramos que tener evidencia empírica sobre su impacto real en la práctica y en el aprendizaje de los estudiantes debería ser prioritario para los investigadores en DPD de Singapur.

Un estudio reciente realizado por Lim, Lee, Saito y Syed Haron (2011) ha mostrado que los profesores de Singapur suelen tener actitudes positivas hacia "lesson study", pues consideran que les ofrece la oportunidad de aprender de sus colegas y de crecer profesionalmente. Los participantes del estudio manifestaron que este enfoque de DPD tuvo un impacto positivo en sus estrategias de enseñanza y que les ayudó a desarrollar un mejor conocimiento de sus estudiantes. Lim et al. (2011) también encontraron que los profesores que implementaron lecciones de investigación por sí mismos tenían actitudes incluso más positivas hacia "lesson study". Esta investigación puso de relieve una serie de factores esenciales para el éxito de "lesson study", como contar con el apoyo de los líderes de la escuela, la propia convicción de los profesores acerca de la eficacia de este enfoque, la provisión de tiempo incluido en sus horarios semanales, y la presencia de un facilitador especializado en "lesson study" dentro del grupo de trabajo.

\section{Desafíos}

Pese a la gran cantidad de recursos ofrecidos para apoyar el desarrollo profesional de los docentes en Singapur, también existen desafíos y limitaciones importantes a considerar. Uno de ellos tiene que ver con la accesibilidad. Por ejemplo, a los profesores de algunas escuelas primarias sólo se les permite asistir a actividades de DPD relacionadas con su especialidad principal (p.ej., matemáticas), pese a que la mayoría imparten diferentes asignaturas (p.ej., música, artes). Ello les hacer perder oportunidades que les permitirían mejorar sus competencias docentes en otras áreas que también enseñan (Wong y Bautista, en revisión). Un segundo reto tiene que ver con la abrumadora cantidad de trabajo de los profesores (Hairon y Dimmock, 2012), incluyendo no sólo carga docente sino también diversas responsabilidades 
académicas (p.ej., evaluaciones, reuniones con padres) y tareas administrativas (p.ej., sesiones en comités). El cumplimiento de todas estas responsabilidades dificulta a los profesores poner toda su energía, compromiso y entusiasmo en las actividades de DPD en las que participan.

Otro desafío está relacionado con los exámenes clasificatorios. El sistema educativo de Singapur es altamente competitivo y selectivo (Hogan y Gopinathan, 2008). La obtención de buenas calificaciones es esencial para garantizar el futuro profesional de los estudiantes. Al igual que en otros países de Asia, ser exitoso académicamente es altamente valorado y recompensado por las familias y por la sociedad en general. Además, las calificaciones de los estudiantes en los exámenes clasificatorios constituye uno de los factores considerados en las evaluaciones del desempeño docentes. Por todas estas razones, muchos educadores en Singapur sienten la necesidad de "enseñar para el examen" y de "machacar a los estudiantes" con clases expositivas y con ejercicios de naturaleza reproductiva, dejando atrás las herramientas que han adquirido en las actividades de DPD (p.ej., métodos de enseñanza innovadores, pedagogías centradas en el estudiante, actividades de aprendizaje basadas en preguntas). Por otra parte, los profesores se enfrentan a menudo con intensas presiones por parte de los padres, quienes les piden que recurran a la enseñanza memorística para asegurar que sus hijos obtengan buenas calificaciones en los exámenes. Por ejemplo, algunos padres se quejan cuando la cantidad de tareas asignada por un profesor es baja, pidiendo más y más deberes para sus hijos. Este tipos de presiones parentales son también lo suficientemente potentes como para limitar el impacto de las actividades de DPD en las prácticas de aula, además de para generar frustración en los docentes y directores de centros educativos (Berthelsen, Brownlee y Karuppiah, 2011).

\section{Sumario y Conclusiones}

"La enseñanza tiene que ser, ante todo, una profesión de aprendizaje; los profesores tendrán que aprender a aprender de diferentes maneras, y a reinventarse a sí mismos como especialistas avanzados del aprendizaje, con sus alumnos como aprendices" [Teaching has to become, first and foremost, a learning profession; teachers will have to learn to learn in different ways, and reconstruct themselves as advanced specialist practitioners of learning with their pupils as their apprentices]. (Tripp, 2002, p. 4)

En este trabajo, hemos descrito el panorama de oportunidades de DPD que se ofrecen a los profesores de escuelas primarias y secundarias del MOE en Singapur. Hemos visto que la provisión de horas de DPD es extraordinariamente alta (hasta 100 horas voluntarias por año), y que existe una amplia variedad de actividades en que los profesores pueden participar, comprendiendo desde prácticas de DPD formales y estructuradas (ofrecidas principalmente por el NIE, y en menor medida por las Academias de profesores) hasta prácticas innovadoras de carácter más informal 
(realizadas en las Academias y también presentes en el trabajo cotidiano de las escuelas). Las políticas educativas actuales, como el "Modelo de Crecimiento Docente" (2012), velan por un DPD coherente con y sensible a las necesidades e intereses de los profesores. Por ejemplo, normalmente se ofrecen diferentes oportunidades de DPD a profesores con diferentes niveles de experiencia (principiante, avanzado, experto) y con diferentes perfiles profesionales (enseñanza, liderazgo, monitorización especializada). Gran parte de las actividades de DPD se centran en contenidos específicos (p.ej., matemáticas, ciencias, música) y proporcionan a los profesores oportunidades para participar en redes de aprendizaje profesional, beneficiándose así del intercambio colegial y de la colaboración. De hecho, recientemente se ha requerido a todas las escuelas de Singapur adoptar el marco de las Comunidades de Aprendizaje Profesional (CAP), con el fin de promover altos niveles de participación colectiva entre los profesores y líderes dentro de cada institución escolar.

Concluimos que este amplio abanico de recursos de DPD, considerados en conjunto, presenta las características del DPD de "alta calidad" descrito en la literatura internacional (Avalos, 2011; Bautista et al, 2015; Borko, 2004; DarlingHammond y McLaughlin, 2011; Desimone, 2009). En particular, el modelo actual de DPD en Singapur: (a) se centra en contenidos escolares específicos y está directamente conectado con la realidad del aula; (b) es intensivo (larga duración) y constante (los profesores participante en DPD todos los años); (c) proporciona a los profesores oportunidades para el aprendizaje activo; (d) promueve la participación colectiva entre los profesores, a través de y dentro de las escuelas; y (e) es coherente con las necesidades e intereses de los docentes y con las prioridades escolares y nacionales. No obstante, pese a todos los aspectos positivos discutidos, también hemos sugerido que es necesario realizar investigación más sistemática, que permita determinar en qué medida este comprensivo y ambicioso modelo de DPD está ayudando a los profesores a mejorar aún más su conocimiento y su práctica, y en última instancia el aprendizaje de los estudiantes (Hill et al., 2013).

Como dijimos en la Introducción, somos conscientes de que el éxito del sistema educativo de Singapur no puede ser explicado exclusivamente por la calidad y cantidad del DPD ofrecido a su personal docente, pues existen numerosos factores de nivel macro, organizacional y familiar que son importantes en esta ecuación (Dimmock y Tan, 2013; Hairon y Dimmock, 2011; Tan y Dimmock, 2014). Con esta precaución en mente, el presente artículo muestra que el sistema educativo de Singapur ha respondido muy bien a los retos de las reformas curriculares y pedagógicas. Los recursos han sido más que adecuados y la variedad de oportunidades de DPD se han convertido en la norma. Los generosos sistemas de permiso y el patrocinio del MOE han permitido a muchos profesores participar en actividades de DPD (incluyendo en programas de postgrado), favoreciendo el desarrollo de nuevos conocimientos y habilidades. Ha habido un claro reconocimiento de que apoyar el desarrollo profesional de los docentes es esencial, de que la enseñanza debe ser concebida y practicada como una profesión reflexiva, y de que los profesores han de ser responsables de su propio crecimiento profesional 
(Deng et al., 2013). Invitamos a los lectores de la revista Psychology, Society and Education a considerar cómo las ideas presentadas en este artículo podrían ser utilizadas para mejorar los modelos de DPD en sus propios países.

\section{Referencias}

Avalos, B. (2011). Teacher professional development in teaching and teacher education over ten years. Teaching and Teacher Education, 27(1), 10-20.

Bautista, A., Cañadas, M. C., Brizuela, M. B., \& Schliemann, A. D. (2015). Examining how teachers use graphs to teach mathematics in a professional development program. Journal of Education and Training Studies, 3(2), 91-106. http://redfame.com/journal/index.php/jets/article/view/676/624

Bautista, A., Ortega-Ruíz, R. (2015). Teacher professional development: International perspectives and approaches. Psychology, Society and Education, 7(3), 240-251.

Berthelsen, D., Brownlee, J., \& Karuppiah, N. (2011). Teaching beliefs and practices in early childhood education in Singapore Singapore: Pearson Education.

Borko, H. (2004). Professional development and teacher learning: Mapping the terrain. Educational Researcher, 33(8), 3-15.

Chong, T. H., \& Fong, H. K. (2000). Pre-service preparation and professional development of mathematics teachers in Singapore. The Mathematics Educator, 5(1/2), 83-90.

Costes-Onishi, P., \& Caleon, I. (2014). Generalists to specialists: Transformative evidences on the practices of music and art teachers in Singapore. Paper presented at the AARE-NZARE International Conference, Brisbane, Australia.

Darling-Hammond, L. (2010). Teaching for deeper learning: Developing a thinking pedagogy In A. P. C. Avila, C. Hui, J. H. Lin, J. C. Peng Tam \& J. C. Lim (Eds.), Rethinking Educational Paradigms: Moving from Good to Great. CJ Koh Professorial Lecture Series No. 5 (pp. 13-18). Singapore: Office of Education Research, National Institute of Education.

Darling-Hammond, L., \& McLaughlin, M. W. (2011). Policies that support professional development in an era of reform. Phi Delta Kappan, 92(6), 81-95.

Deng, Z., Gopinathan, S., \& Lee, C. K.-E. (2013). Globalization and the Singapore curriculum: From policy to classroom. Singapore: Springer.

Desimone, L. M. (2009). Improving impact studies of teachers' professional development: Toward better conceptualizations and measures. Educational Researcher, 38(3), 181199.

Dimmock, C., \& Tan, C. Y. (2013). Educational leadership in Singapore. Journal of Educational Administration, 51(3), 320-340. doi: http://dx.doi.org/10.1108/09578231311311492

Ellis, N. J. (2014). Afraid to lose out: the impact of kiasuism on practitioner research in Singapore schools. Educational Action Research, 22(2), 235-250. doi: $10.1080 / 09650792.2013 .859088$

Ellis, N. J., \& Armstrong, A. C. (2013). How context shapes practitioner research and professional learning in schools in Singapore and NSW. Compare: A Journal of Comparative and International Education, 44(3), 435-454. doi: $10.1080 / 03057925.2013 .767674$ 
Goh, C. T. (1997). Speech by Prime Minister Goh Chok Tong at the opening of the 7th International Conference on Thinking", Monday, June 2, 1997, at 9.00 am at the Suntec City Convention Centre Ballroom. Available at http://www.moe.gov.sg/media/speeches/1997/020697.htm.

Gopinathan, S. (2012). Fourth way in action? The evolution of Singapore's education system. Educational Research for Policy and Practice, 11(1), 65-70. doi: http://dx.doi.org/10.1007/s10671-011-9117-6

Hairon, S. (2006). Action research in Singapore education: constraints and sustainability. Educational Action Research, 14(4), 513-523. doi: 10.1080/09650790600975684

Hairon, S., \& Dimmock, C. (2011). Singapore schools and professional learning communities: teacher professional development and school leadership in an Asian hierarchical system. Educational Review, 64(4), 405-424. doi: $10.1080 / 00131911.2011 .625111$

Heong, Y. L. (2012). Lesson study as professional development of science teachers in professional learning community schools. SCIOS, 48(1), 4.

Hill, H. C., Beisiegel, M., \& Jacob, R. (2013). Professional development research: Consensus, crossroads, and challenges. Educational Researcher, 42(9), 476-487.

Hogan, D., \& Gopinathan, S. (2008). Knowledge management, sustainable innovation, and preservice teacher education in Singapore. Teachers \& Teaching, 14(4), 369-384.

Kaur, B. (2011). Enhancing the pedagogy of mathematics teachers (EPMT) project: a hybrid model of professional development. ZDM, 43(6-7), 791-803. doi: 10.1007/s11858-011-0364-z

Kazemi, E., \& Hubbard, A. (2008). New directions for the design and study of professional development: Attending to the coevolution of teachers' participation across contexts. Journal of Teacher Education, 59(5), 428-441.

Koh, K. H. (2011). Improving teachers' assessment literacy through professional development. Teaching Education 22(3), 255-276.

Lee, D., Hong, H., Tay, W., \& Lee, W. O. (2013). Singapore Professional Learning Communities in Singapore Schools. UK Society for Co-operative Studies, 46(2), 5356.

Lewis, C., Perry, R., \& Hurd, J. (2004). A deeper look at lesson study. Educational Leadership, 61(5), 18-23.

Lim, C., Lee, C., Saito, E., \& Syed Haron, S. (2011). Taking stock of Lesson Study as a platform for teacher development in Singapore. Asia-Pacific Journal of Teacher Education, 39(4), 353-365. doi: 10.1080/1359866X.2011.614683

Ministry of Education (MOE). (2005a). Speech by Mr Tharman Shanmugaratnam, Minister for Education, at the MOE Work Plan Seminar 2004, Ngee Ann Polytechnic Convention Centre, Thursday 22 September, 10:00 a.m. http://www.moe.gov.sg/media/speeches/2005/sp20050922.htm

Ministry of Education (MOE). (2005b). Teach Less, Learn More. Singapore Education Milestones 2004-2005. Singapore: Retrieved from http://moeedu.sg/about/yearbooks/2005/teach.html.

Ministry of Education (MOE). (2010). Schools as professional learning communities. Singapore: Author.

Ministry of Education (MOE). (2012). The Teacher Growth Model. Singapore: Retreived from http://www.moe.gov.sg/media/press/files/2012/05/fact-sheet-teacher-growthmodel.pdf. 
National Institute of Education (NIE). (2009). A Teacher Education Model for the 21st Century. Singapore: NIE.

OECD. (2014). A Teachers' Guide to TALIS 2013: Teaching and Learning International Survey. Paris: TALIS, OECD Publishing.

Schwab, K. (2015). World Economic Forum's Global Competitiveness Report, 2014-2015. Retrived from: http://reports.weforum.org/global-competitiveness-report-20142015/. Switzerland: World Economic Forum.

Tan, C. Y., \& Dimmock, C. (2014). How a 'top-performing'Asian school system formulates and implements policy: the case of Singapore. Educational Management Administration \& Leadership, 1741143213510507.

Tan, I., Wong, I., \& Goh, C. (2010). Strategic teacher professional development. In A. Y. Chen \& S. L. Koay (Eds.), Transforming Teaching, Inspiring Learning. 60 years of teacher education in Singapore (1950-2010) (pp. 147-158). Singapore: NIE/NTU.

Tan, Y., \& Nashon, S. (2013). Promoting Teacher Learning Through Learning Study Discourse: The Case of Science Teachers in Singapore. Journal of Science Teacher Education, 24(5), 859-877. doi: 10.1007/s10972-013-9340-5

Tan, Y. M. (2014). Enriching a collaborative teacher inquiry discourse: exploring teachers' experiences of a theory-framed discourse in a Singapore case of lesson study. Educational Action Research, 22(3), 411-427. doi: 10.1080/09650792.2014.880360

Training and Development Division (TDD). (2010). Schools as professional learning communities. Singapore: Training and Development Division, Ministry of Education.

Tripp, D. (2002). Valuing the teaching profession: purpose, passion and hope. Response to Margot Cameron-Jones' and Belinda Charles' plenary papers presented at the Singapore National Institute of Education Symposium. Openning of Nanyang Campus, 25 January. Available on VCD from NIE (Singapore).

Tripp, D. (2004). Teachers' networks: A new approach to the professional development of teachers in Singapore. In C. Day \& J. Sachs (Eds.), International handbook on the continuing professional development of teachers (pp. 191-214). Maidenhead England: Open University Press.

Wang, X., Kim, B., Lee, J. W. Y., \& Kim, M. S. (2014). Encouraging and being encouraged: Development of an epistemic community and teacher professional growth in a Singapore classroom. Teaching and Teacher Education, 44, 12-24. doi: http://dx.doi.org/10.1016/j.tate.2014.07.009

Wong, K. Y. (2013). Diverse pathways for life-long teacher professional development. Paper presented at the International Science, Mathematics and Technology Education Conference (ISMTEC 2013), Bangkok, Thailand.

Wong, J., \& Bautista, A. (submitted). Features of most and least helpful professional development experiences: A study with primary music specialists. Paper presentation. Annual Meeting of AERA, Public Scholarship to Educate Diverse Democracies. Washington, DC (April, 2016). United States. 